\title{
Alternativas de compostaje de aserrín de pino caribe (Pinus caribaea) en la industria maderera Refocosta S.A., municipio de Villanueva, Casanare, Colombia \\ Alternatives for composting Caribbean Pine sawdust (Pinus caribaea) in the wood industry Refocosta S. A., in the municipality of Villanueva, Casanare, Colombia
}

\author{
Mónica Bibiana Sarmiento Oviedo \\ E-mail: mobisato@hotmail.com \\ Universidad Nacional del Centro del Perú
}

Resumen.- Debido a la actual acumulación de aserrín, subproducto del proceso del aserrado de la especie Pinus caribaea en la empresa maderera Refocosta S. A., se ensayó el compostaje en tres tratamientos con diferentes fuentes nitrogenadas (urea, lodos industriales de aceite de palma y estiércol bovino) y se comparó con corteza de pino compostada y aserrín expuesto a la intemperie. Para evaluar la posible inhibición de los sustratos, se midió germinación y biomasa fresca en Zea maíz y Acacia mangium. Se trasplantaron plántulas de Pinus caribaea, Eucalyptus pellita y Acacia mangium y a los dos meses se evaluó longitud del tallo, vigor y supervivencia. Se presentaron diferencias entre maíz y Acacia mangium y se observó menor germinación y biomasa en los sustratos aserrín a la intemperie y corteza sin compostar. El vigor y la longitud del tallo fue significativamente mejor en corteza compostada, sustrato actualmente utilizado para la producción de plántulas en el vivero de Refocosta; los sustratos a base de aserrín presentaron deficiencias marcadas (coloraciones rojizas, necrosis y enanismo).

Palabras clave: Compostaje de aserrín, corteza compostada de Pinus caribaea, fuentes de nitrógeno.

Abstract.- Given the day-to-day accumulation of sawdust, a byproduct of the milling process of the Pinus caribaea species in the Wood industry of Refocosta, a composting process was tested in three treatments with different nitrogen sources (urea, industrial palm oil sludge, cattle dung) and it was compared with composted pine bark and sawdust exposed to the weather. To assess possible inhibition of the substrates, the fresh biomass and germination of Zea maize and Acacia mangium was measured. Seedlings of Pinus caribaea, eucalyptus Pellita and Acacia Mangium were transplanted, and two months later the stem length, strength and survival were assessed. There were differences between maize and Acacia mangium and a lower germination and biomass in the substrates, sawdust and bark exposed to the weather and without composting were observed. The vigor and stem length were significantly better in composted bark, substrate which is currently used in the 
vivarium of Refocosta to produce seedlings; the sawdust-based substrates showed marked deficits (red colors, necrosis and stunting).

Key words: Sawdust compost, Pinus caribaea composted bark, composting, nitrogen sources.

\section{Introducción}

El cambio de vegetación tipo sabana a plantaciones forestales (de palma, caucho y pino) constituye uno de los rasgos del proceso de expansión de la frontera agrícola en la región de los llanos orientales de Colombia, promovido por el gobierno nacional en el marco de la política de las cadenas productivas.

En el desarrollo de la cadena forestal de pino en la región se destacan los proyectos Gaviotas en la altillanura del Vichada, con aproximadamente 8.000 ha de Pinus caribaea destinados a la producción de colofonia y Reforestadora de la Costa S. A., en Villanueva, Casanare, con 3500 ha, destinada a la producción de madera rolliza, aserrada, seca y de segunda transformación.

En los últimos años el mejoramiento y la automatización de nuevos equipos de aserrado en Reforestadora de la Costa (Refocosta) contribuyeron a incrementar en más de $50 \%$ la producción de madera aserrada, incluyendo nuevas líneas (vigas laminadas, tableros, finger joint). Sin embargo, también se incrementó la cantidad de residuos de aserrado, costos de movilización, riesgos de incendio y problemas sanitarios derivados de estos.

En Refocosta el aprovechamiento del proceso de tala y troceo es del $75 \%$; de aserrado de primera transformación, de150.4\% y en segunda transformación, del $57.7 \%$. La cantidad promedio generada de residuos tipo aserrín y viruta es de $235.2 \mathrm{~m}^{3}$ (Reforestadora de la costa S. A. 2008).

Aunque los residuos tipo orillo o trozos pequeños de madera previamente astillados se utilizan como combustible para la caldera de secado de la madera, y el aserrín y/o viruta cuentan con compradores que los utilizan para galpones, porquerizas o establos, el volumen de venta es inferior al producido $\left(36.9 \mathrm{~m}^{3} / \mathrm{mes}\right)$.

El Centro de Estudios de Biomasa Forestal de la Universidad de Pinar del Río de Cuba afirma que los residuos forestales como el aserrín son altamente contaminantes para el medio ambiente, mientras que la Organización de las Naciones Unidas para la Agricultura y la Alimentación -FAO- advierte sobre el impacto del aserrín como agente contaminante del suelo y del agua (Álvarez et al., 2004).

La lenta degradación natural de los residuos, la reducción del espacio disponible en los centros de elaboración de madera, el detrimento a la salud de los trabajadores y vecinos de los aserraderos, los riesgos ambientales por incendios y autocombustión y las condiciones para la propagación de plagas y enfermedades (especialmente hongos de los géneros Fomes, Schyzophylum y Polyporus), amerita centrar la atención en alternativas 
de manejo con menos repercusiones negativas para el ambiente y que generen valor agregado para la actividad reforestadora.

El objetivo de la investigación consistió en examinar alternativas de compostaje del aserrín derivado del procesamiento industrial del pino caribe (Pinus caribaea) de Refocosta para utilizarlo como material orgánico acondicionador del suelo estable y seguro en procesos de cultivo asociados a las cadenas agrícolas de la región.

\section{Materiales y Métodos}

El estudio se desarrolló en la empresa Reforestadora de La Costa S. A. (4 ${ }^{\circ}$ $39^{\prime} 06.94 \mathrm{~N}$ y $72^{\circ} 54^{\prime} 59.60 \mathrm{~W}, 25.8^{\circ} \mathrm{C}$ de temperatura media anual, $2911 \mathrm{~mm}$ de precipitación anual), localizada en el municipio de Villanueva, Casanare, Colombia, a $220 \mathrm{~km}$ de la capital del país. Según la clasificación de zonas de vida de Holdridge, corresponde a bosque húmedo tropical (bh-T) situado en terraza alta originada por deposiciones de sedimentos de los ríos Upía y Tua.

Los suelos presentan textura franco arenosa, son muy lavados, con bajo $\mathrm{pH}$, contenido alto de aluminio, pobres en fósforo, nitrógeno y materia orgánica y por tanto, acusan baja fertilidad; el contenido de arcilla caolinítica aumenta con la profundidad por intemperización del cuarzo dominante en el material de origen (Bongcam, 1982).

Se diseñaron cuatro tratamientos con aserrín fresco y fuentes nitrogenadas, apilado en líneas de longitud variable, altura de $1.5 \mathrm{~m}$. y ancho de $3 \mathrm{~m}$., a la intemperie (Testigo), con estiércol bovino recolectado de corrales y bebederos en proporción $3: 1$, con lodos (de aguas tratadas de la agroindustria de aceite de palma) en dosis de $75 \mathrm{l} / \mathrm{m}^{3}$ y con $5 \mathrm{~kg}$ de urea $/ \mathrm{m}^{3}+7 \mathrm{cc} / \mathrm{m}^{3}$ de compuesto bacterial (Tabla 1).

Para favorecer condiciones aerobias, los tratamientos fueron humedecidos $\mathrm{y}$ volteados semanalmente con el equipo Aeromaster durante dos meses y se realizaron mediciones periódicas de temperatura y $\mathrm{CO} 2$.

Se realizaron análisis químicos (Contenido de N, P, K, Ca, Mg, S, medidos en porcentaje y $\mathrm{Fe}, \mathrm{Cu}, \mathrm{Mn}, \mathrm{Zn}$, $\mathrm{B}$, medidos en $\mathrm{mg} / \mathrm{Kg}$ ) a cada tratamiento en el laboratorio de suelos de Corpoica y se compararon igualmente con compost de corteza de pino que Refocosta viene utilizando hace tres años como sustrato para la producción en vivero de material clonal y por semilla. Se realizó análisis de costos de los tratamientos para considerar la viabilidad económica de las alternativas.

\section{Pruebas de germinación}

Mediante diseño estadístico totalmente al azar, los tratamientos y el sustrato tradicional empleado en vivero (corteza compostada), se sometieron en tres repeticiones a pruebas de germinación de maíz (Zea maíz) y Acacia mangium. Se colocaron 30 semillas de maíz por cajón de madera y 100 de acacia sin fertilización. Las variables a medir fueron $\%$ de germinación y biomasa fresca al mes (maíz) y a las dos semanas (Acacia). 
Pruebas de crecimiento

Se estableció prueba de crecimiento en tres especies (Pinus caribaea, Eucalyptus pellita y Acacia mangium). Los plantines fueron germinados previamente $\mathrm{y}$ trasladados a bandejas de 40 tubetes, cada una de las cuales constituyó una réplica. Los sustratos no se fertilizaron pero las fertilizaciones de mantenimiento se realizaron foliarmente aplicando elementos mayores y menores.
A las seis semanas se hicieron mediciones de las variables longitud del tallo y supervivencia y se evaluó cualitativamente el desarrollo (Calificación de 0: plántula con desarrollo inferior al normal, baja lignificación, deficiencias nutricionales y sanitarias; 1: plántula con desarrollo promedio con leves síntomas de deficiencias y 2: plántula con buen vigor, color y desarrollo foliar). Se realizaron análisis de varianza aplicando Pruebas de Duncan.

Tabla 1. Contenido biológico de un litro del producto agregado a pilas compostadas con urea en la Reforestadora del Pacífico S. A.

\begin{tabular}{lc}
\hline \multicolumn{1}{c}{ Microorganismos } & $\begin{array}{c}\text { Unidades Formadoras de } \\
\text { Colonia-UFC/ml }\end{array}$ \\
\hline Azospirillum brasilense & $40 \times 10^{6}$ \\
Azotobacter chrococcum & $30 \times 10^{6}$ \\
Lactobacillus acidophillus & $100 \times 10^{6}$ \\
Saccharomyces cerevisae & 100.000 \\
\hline
\end{tabular}

\section{Resultados}

\section{Análisis químico}

El contenido de elementos mayores fue bajo y no se encontraron diferencias notorias entre sustratos, mientras que los elementos menores presentaron variaciones considerables, especialmente respecto al aserrín a la intemperie, posiblemente debidos al proceso de compostación y a la adición de materias primas (Tabla 2).

Tabla 2. Análisis químico del aserrín de pino Caribe compostado en REFOCOSTA bajo diferentes tratamientos

\begin{tabular}{|l|c|c|c|c|c|c|c|c|c|c|c|c|}
\hline \multicolumn{1}{|c|}{ Tratamiento } & N & P & K & Ca & Mg & S & Fe & Cu & Mn & Zn & B \\
\hline & \multicolumn{9}{|c|}{ \% } \\
\hline Aserrín a la intemperie & 0,3 & 0,08 & 0,04 & 0,07 & 0,02 & 0,01 & 233 & 1161 & 45 & 7 & 32 \\
\hline Sustrato de corteza de pino & 1,2 & 0,02 & 0,05 & 0,09 & 0,02 & 0,03 & 4242 & 3 & 73 & 11 & 1 \\
\hline $\begin{array}{l}\text { Aserrín compostado con } \\
\text { estiércol bovino }\end{array}$ & 0,2 & 0,07 & 0,03 & 0,13 & 0,05 & 0,03 & 9725 & 79 & 95 & 53 & 5 \\
\hline $\begin{array}{l}\text { Aserrín compostado con lodos } \\
\text { residuales }\end{array}$ & 0,3 & 0,02 & 0,02 & 0,09 & 0,04 & 0,01 & 1754 & 4 & 116 & 7 & 3 \\
\hline Aserrín compostado con urea & 0,2 & 0,01 & 0,01 & 0,07 & 0,01 & 0,01 & 3262 & 13 & 130 & 11 & 5 \\
\hline
\end{tabular}




\section{Análisis de costos}

El compostaje de corteza es el de mayor costo debido al alto precio y cantidad del insumo que utiliza como fuente nitrogenada y a que se requiere un proceso y maquinaria especializada para obtenerlo (descortezado mecánico). Los tratamientos restantes derivaron los costos principalmente de la movilización y recolección de sus materias primas; en la medida que estas empiecen a tener un costo se elevará ostensiblemente el valor final del metro cúbico. En el caso del aserrín con urea, el mayor costo se origina de dicho fertilizante, pero debido a su baja dosificación comparada con la corteza, su valor final se encuentra dentro del rango de los otros sustratos que utilizan este residuo como base (Tabla 3).

Tabla 3. Costos operacionales para el compostaje de $1 \mathrm{~m}^{3}$ de residuos de serrado de pino caribe bajo diferentes tratamientos en Refocosta

\begin{tabular}{|c|c|c|c|c|c|c|c|c|c|c|c|}
\hline Tratamiento & $\begin{array}{c}\text { Recolección } \\
\text { de Estiércol } \\
\text { (Jornal) }\end{array}$ & $\begin{array}{c}\text { Transporte } \\
\text { de estiércol } \\
\text { en Tractor. } \\
\text { (Hora/ } \\
\text { máquina) }\end{array}$ & $\begin{array}{l}\text { Urea } \\
(\mathrm{Kg})\end{array}$ & $\begin{array}{c}\text { Transporte } \\
\text { de lodos en } \\
\text { tractor } \\
\text { (Hora/ } \\
\text { máquina) }\end{array}$ & $\begin{array}{l}\text { Lodos } \\
\left(\mathbf{m}^{3}\right)\end{array}$ & $\begin{array}{c}\text { Estiércol } \\
\left(\mathbf{m}^{3}\right)\end{array}$ & $\begin{array}{c}\text { Aserrín } \\
\left(\mathrm{m}^{3}\right)\end{array}$ & $\begin{array}{c}\text { Corteza } \\
\left(\mathbf{m}^{3}\right)\end{array}$ & $\begin{array}{c}\text { Bacterias } \\
\text { (L) }\end{array}$ & $\begin{array}{c}\text { Volteo e } \\
\text { hidratación } \\
\text { (Hora/ } \\
\text { máquina) }\end{array}$ & Total \\
\hline \$ unitario & 30.000 & 25.000 & 1.500 & 25.000 & - & - & 2.000 & 5.000 & 42.000 & 31.250 & \\
\hline $\begin{array}{l}\text { Testigo 1: } \\
\text { Aserrín a la } \\
\text { intemperie }\end{array}$ & 0 & 0 & 0 & 0 & 0 & 0 & 1 & 0 & 0 & 0.17 & 7.357 \\
\hline $\begin{array}{l}\text { Testigo 2: } \\
\text { Sustrato de } \\
\text { corteza de } \\
\text { pino }\end{array}$ & 0 & 0 & 10 & 0 & 0 & 0 & 0 & 1 & 0.007 & 0.17 & 25.651 \\
\hline $\begin{array}{l}\text { Tratamiento } \\
\text { 1: Aserrín } \\
\text { compostado } \\
\text { con estiércol } \\
\text { de bovino }\end{array}$ & 0.17 & 0.17 & 0 & 0 & 0 & 0.33 & 1 & 0 & 0 & 0.17 & 16.786 \\
\hline $\begin{array}{l}\text { Tratamiento } \\
\text { 2: Aserrín } \\
\text { compostado } \\
\text { con lodos } \\
\text { residuales }\end{array}$ & 0 & 0 & 0 & 0.23 & 0.075 & 0 & 1 & 0 & 0 & 0.17 & 13.071 \\
\hline $\begin{array}{l}\text { Tratamiento } \\
\text { 3: Aserrín } \\
\text { compostado } \\
\text { con urea }\end{array}$ & 0 & 0 & 5 & 0 & 0 & 0 & 1 & 0 & 0.007 & 0.17 & 15.151 \\
\hline
\end{tabular}

\section{Pruebas de germinación}

En maíz los tratamientos de aserrín con urea estiércol y lodos residuales presentan diferencias significativas respecto a corteza compostada y aserrín a la intemperie aunque no presentan diferencias significativas entre sí. Los sustratos utilizados para la geminación de semillas de Acacia mangium no presentaron diferencias significativas (Tablas 4 y 5). 
Tabla 4. Anava de \% de germinación de maíz y Acacia mangium en condiciones de Refocosta

\begin{tabular}{lllllll}
\hline Especie & \multicolumn{1}{c}{ FV } & GL & SC & CM & \multicolumn{1}{c}{ F } & \\
\hline Zea maiz & Tratamientos & 4 & 2444.9 & 611.23 & 30.561667 & $* *$ \\
& Error & 10 & 200.0 & 20 & & \\
& Total & 14 & 2644.9 & & & \\
\hline Acacia mangium & Tratamientos & 4 & 153.7 & 38.433 & 1.37619 & NS \\
& Error & 10 & 280.0 & 28 & & \\
& Total & 14 & 433.7 & & & \\
\hline
\end{tabular}

Tabla 5. Prueba de Duncan \% de germinación de maíz

\begin{tabular}{lcccc}
\hline $\begin{array}{c}\text { Prueba de } \\
\text { diferencias }\end{array}$ & $\begin{array}{c}\text { Diferencia } \\
\text { encontrada }\end{array}$ & & RMS & Significancia \\
\hline E-D & 6 & $<$ & 11.6 & NS \\
E-C & 12 & $<$ & 12.2 & NS \\
E-B & 14 & $>$ & 12.6 & $* *$ \\
E-A & 37 & $>$ & 12.8 & $* *$ \\
D-C & 6 & $<$ & 12.2 & NS \\
D-B & 8 & $<$ & 12.6 & NS \\
D-A & 31.7 & $>$ & 12.8 & $* *$ \\
C-B & 2.3 & $<$ & 12.6 & NS \\
C-A & 25.7 & $>$ & 12.8 & $* *$ \\
B-A & 23.3 & $>$ & 12.6 & $* *$ \\
\hline
\end{tabular}

Corteza: A; Aserrín: B; Aserrín + urea: C; Aserrín + estiércol: D; Aserrín + lodo: E.

\section{Biomasa fresca}

En maíz el tratamiento aserrín con lodos fue significativamente mejor para la producción de biomasa fresca; aserrín con estiércol solo presentó diferencias significativas con el tratamiento de menor valor (corteza). En Acacia mangium, entre los tratamientos aserrín con lodos y aserrín con estiércol no se presentaron diferencias significativas (Tablas 6 y 7 ). 
Tabla 6. Anava para Biomasa fresca en Maíz y Acacia mangium

\begin{tabular}{|c|l|r|r|r|r|r|}
\hline Especie & \multicolumn{1}{|c|}{ FV } & GL & SC & CM & F & \\
\hline \multirow{5}{*}{ Zea maiz } & Tratamientos & 4 & 7.4 & 1.8488 & 30.561667 & $* *$ \\
\cline { 2 - 8 } & Error & 10 & 1.3 & 0.1302 & & \\
\cline { 2 - 8 } & Total & 14 & 8.7 & & & \\
\hline \multirow{2}{*}{$\begin{array}{l}\text { Acacia } \\
\text { mangium }\end{array}$} & Tratamientos & 4 & 384.9 & 96.228 & 168.62325 & $* *$ \\
\cline { 2 - 8 } & Error & 10 & 5.7 & 0.5707 & & \\
\cline { 2 - 7 } & Total & 14 & 390.6 & & & \\
\hline
\end{tabular}

Tabla 7. Prueba de Duncan para Biomasa fresca en Maíz y Acacia mangium

\begin{tabular}{|c|c|c|c|c|c|c|c|c|}
\hline \multicolumn{5}{|c|}{ Zea maíz } & \multicolumn{4}{|c|}{ Acacia mangium } \\
\hline $\begin{array}{l}\text { Prueba de } \\
\text { diferencias }\end{array}$ & $\begin{array}{l}\text { Diferencia } \\
\text { encontrada }\end{array}$ & & RMS & Significancia & $\begin{array}{l}\text { Diferencia } \\
\text { encontrada }\end{array}$ & & RMS & Significancia \\
\hline E-D & 1 & $>$ & & $* *$ & 7.5 & $>$ & 2.0 & $* *$ \\
\hline E-C & 1 & $>$ & & $* *$ & 7.9 & $>$ & 2.1 & $* *$ \\
\hline E-B & 2 & $>$ & & $* *$ & 11.6 & $>$ & 2.1 & $* *$ \\
\hline E-A & 2 & $>$ & & $* *$ & 15.2 & $>$ & 2.2 & $* *$ \\
\hline D-C & 0 & $<$ & & NS & 0.4 & $<$ & 2.1 & NS \\
\hline D-B & 1 & $<$ & & NS & 4.1 & $>$ & 2.1 & $* *$ \\
\hline D-A & 1.1 & $>$ & & $* *$ & 7.7 & $>$ & 2.2 & $* *$ \\
\hline C-B & 0.4 & $<$ & & NS & 3.7 & $>$ & 2.1 & $* *$ \\
\hline $\mathrm{C}-\mathrm{A}$ & 0.7 & $<$ & & NS & 3.7 & $>$ & 2.2 & $* *$ \\
\hline B-A & 0.4 & $<$ & & NS & 3.6 & $>$ & 2.1 & $* *$ \\
\hline \multicolumn{5}{|c|}{$\begin{array}{l}\text { Corteza: A; Aserrín: B; Aserrín + urea: C; Aserrín + } \\
\text { estiércol: D; Aserrín + lodo: E }\end{array}$} & \multicolumn{4}{|c|}{$\begin{array}{l}\text { Aserrín: A; Corteza: B; Aserrín + } \\
\text { estiércol: C; Aserrín + lodos: D; Aserrín } \\
\text { + urea: E. }\end{array}$} \\
\hline
\end{tabular}

\section{Pruebas de crecimiento}

\section{Longitud del tallo}

En Pino caribaea el sustrato de corteza fue significativamente superior a los demás tratamientos. Se presentaron diferencias significativas entre la longitud del tallo de las plántulas sembradas en aserrín con estiércol y las de aserrín a la intemperie. En Eucalipto pellita, fue significativa la diferencia entre el tratamiento con corteza y los demás tratamientos. La longitud del tallo de las plántulas de Acacia mangium sembradas en corteza fue significativamente superior a las plántulas de la misma especie sembradas en otros sustratos (Tablas 8 y 9). 
Tabla 8. Anava para longitud de tallo en las especies Pino Caribe, Eucalipto pellita y Acacia mangium Variable

\begin{tabular}{|l|l|r|r|r|r|r|}
\hline \multicolumn{1}{|c|}{ Especie } & \multicolumn{1}{|c|}{ FV } & GL & SC & CM & \multicolumn{1}{c|}{ F } & \\
\hline \multirow{4}{*}{ Pino caribe } & Tratamientos & 4 & 15.7 & 3.9315 & 28.684555 & $* *$ \\
\cline { 2 - 7 } & Error & 15 & 2.1 & 0.1371 & & \\
\cline { 2 - 7 } & Total & 19 & 17.8 & & & \\
\hline \multirow{4}{*}{ Eucalipto pellita } & Tratamientos & 4 & 475.6 & 118.91 & 68.733636 & $* *$ \\
\cline { 2 - 8 } & Error & 15 & 26.0 & 1.73 & & \\
\cline { 2 - 8 } & Total & 19 & 501.6 & & & \\
\hline \multirow{5}{*}{ Acacia mangium } & Tratamientos & 4 & 1444.9 & 361.22 & 889.372 & $* *$ \\
\cline { 2 - 7 } & Error & 15 & 6.1 & 0.4061 & & \\
\cline { 2 - 7 } & Total & 19 & 1451.0 & & & \\
\hline
\end{tabular}

Tabla 9. Prueba de Duncan para longitud del tallo en las especies Pino Caribe, Eucalipto pellita y Acacia mangium en Refocosta

\begin{tabular}{|c|c|c|c|c|c|c|c|c|c|c|c|c|}
\hline \multicolumn{5}{|c|}{ Pinus caribaea } & \multicolumn{4}{|c|}{ Eucalipto pellita } & \multicolumn{4}{|c|}{ Acacia mangium } \\
\hline $\begin{array}{l}\text { Prueba de } \\
\text { diferencias }\end{array}$ & $\begin{array}{l}\text { Diferencia } \\
\text { encontrada }\end{array}$ & & RMS & Significancia & $\begin{array}{c}\text { Diferencia } \\
\text { encontrada }\end{array}$ & & RMS & Significancia & $\begin{array}{c}\text { Diferencia } \\
\text { encontrada }\end{array}$ & & RMS & Significancia \\
\hline E-D & 1.2 & $>$ & 0.8 & $* *$ & 12.3 & $>$ & 2.7 & $* *$ & 21 & $>$ & 1.3 & $* *$ \\
\hline E-C & 1.6 & $>$ & 0.8 & $* *$ & 13.1 & $>$ & 2.9 & $* *$ & 21 & $>$ & 1.4 & $* *$ \\
\hline E-B & 2.0 & $>$ & 0.8 & $* *$ & 13.8 & $>$ & 3.0 & $* *$ & 21 & $>$ & 1.4 & $* *$ \\
\hline E-A & 2.3 & $>$ & 0.8 & $* *$ & 14.0 & $>$ & 3.0 & $* *$ & 21 & $>$ & 1.5 & $* *$ \\
\hline D-C & 0.4 & $<$ & 0.8 & NS & 1 & $<$ & 2.9 & NS & 0.3 & $<$ & 1.4 & NS \\
\hline D-B & 0.8 & $<$ & 0.8 & NS & 2 & $<$ & 3.0 & NS & 1 & $<$ & 1.4 & NS \\
\hline D-A & 1.1 & $>$ & 0.8 & $* *$ & 1.8 & $<$ & 3.0 & NS & 0.6 & $<$ & 1.5 & NS \\
\hline C-B & 0.4 & $<$ & 0.8 & NS & 0.6 & $<$ & 3.0 & NS & 0.2 & $<$ & 1.4 & NS \\
\hline C-A & 0.7 & $<$ & 0.8 & NS & 0.9 & $<$ & 3.0 & NS & 0.3 & $<$ & 1.5 & NS \\
\hline B-A & 0.3 & $<$ & 0.8 & NS & 0.2 & $<$ & 3.0 & NS & 0.1 & $<$ & 1.4 & NS \\
\hline \multicolumn{5}{|c|}{$\begin{array}{l}\text { Aserrín: A; Aserrín + urea: B; Aserrín + lodo: C; Aserrín + } \\
\text { estiércol; D; Corteza: E. }\end{array}$} & \multicolumn{4}{|c|}{$\begin{array}{l}\text { Aserrín + lodos: A; Aserrín: B; Aserrín + urea: } \\
\text { C; Aserrín + estiércol: D; Corteza: E. }\end{array}$} & \multicolumn{4}{|c|}{$\begin{array}{l}\text { Aserrín + urea: A; Aserrín + estiércol: B; } \\
\text { Aserrín: C; Aserrín + lodos: D; Corteza: E. }\end{array}$} \\
\hline
\end{tabular}

\section{Supervivencia}

La supervivencia de las plántulas de Pino caribaea no fue afectada por el uso de ninguno de los sustratos; Eucalipto pellita en sustrato de corteza de pino compostada fue significativamente menor a otros tratamientos, los cuales no presentaron diferencias entre sí (Tablas10 y 11). Los sustratos con Acacia mangium no tuvieron incidencia en la supervivencia de las plántulas. 
Tabla 10. Anava para supervivencia en las especies Pino caribaea, Eucalipto pellita y Acacia mangium

\begin{tabular}{|c|c|c|c|c|c|c|}
\hline Especie & FV & GL & SC & CM & $\mathbf{F}$ & \\
\hline \multirow[t]{3}{*}{ Pino caribaea } & Tratamientos & 4 & 29.0 & 7.25 & 1.208 & NS \\
\hline & Error & 15 & 90.0 & 6 & & \\
\hline & Total & 19 & 119.0 & & & \\
\hline \multirow{3}{*}{$\begin{array}{l}\text { Eucalipto } \\
\text { pellita }\end{array}$} & Tratamientos & 4 & 263.3 & 65.825 & 45.924 & ** \\
\hline & Error & 15 & 21.5 & 1.4333 & & \\
\hline & Total & 19 & 284.8 & & & \\
\hline \multirow{3}{*}{$\begin{array}{l}\text { Acacia } \\
\text { mangium }\end{array}$} & Tratamientos & 4 & 26.7 & 6.675 & 2.9234 & NS \\
\hline & Error & 15 & 34.3 & 2.2833 & & \\
\hline & Total & 19 & 61.0 & & & \\
\hline
\end{tabular}

Tabla 11. Prueba de Duncan para supervivencia en Eucalipto pellita

\begin{tabular}{|l|r|l|r|r|}
\hline $\begin{array}{c}\text { Prueba de } \\
\text { diferencias }\end{array}$ & $\begin{array}{r}\text { Diferencia } \\
\text { encontrada }\end{array}$ & & RMS & Significancia \\
\hline E-D & - & $<$ & 2.5 & NS \\
\hline E-C & 0.3 & $<$ & 2.6 & NS \\
\hline E-B & 2.0 & $<$ & 2.7 & NS \\
\hline E-A & 10.3 & $>$ & 2.7 & $* *$ \\
\hline D-C & 0 & $<$ & 2.6 & NS \\
\hline D-B & 2 & $<$ & 2.7 & NS \\
\hline D-A & 10.3 & $>$ & 2.7 & $* *$ \\
\hline C-B & 1.7 & $<$ & 2.7 & NS \\
\hline C-A & 10.0 & $>$ & 2.7 & $* *$ \\
\hline B-A & 8.3 & $>$ & 2.7 & $* *$ \\
\hline
\end{tabular}

Corteza: A; Aserrín + Estiércol: B; Aserrín + urea: C; Aserrín: D; Aserrín + lodos: E

Vigor

En Pino caribaea, la diferencia entre el tratamiento con corteza y los demás tratamientos empleados fue ostensible, y en plántulas de Eucalipto pellita los resultados cualitativos fueron heterogéneos, encontrándose diferencias altamente significativas entre tratamientos. En Acacia mangium los sustratos de corteza y aserrín con lodos fueron significativamente superiores a los demás tratamientos. Sin embargo, el tratamiento corteza fue muy superior al de lodos (Tablas 12 y 13). 

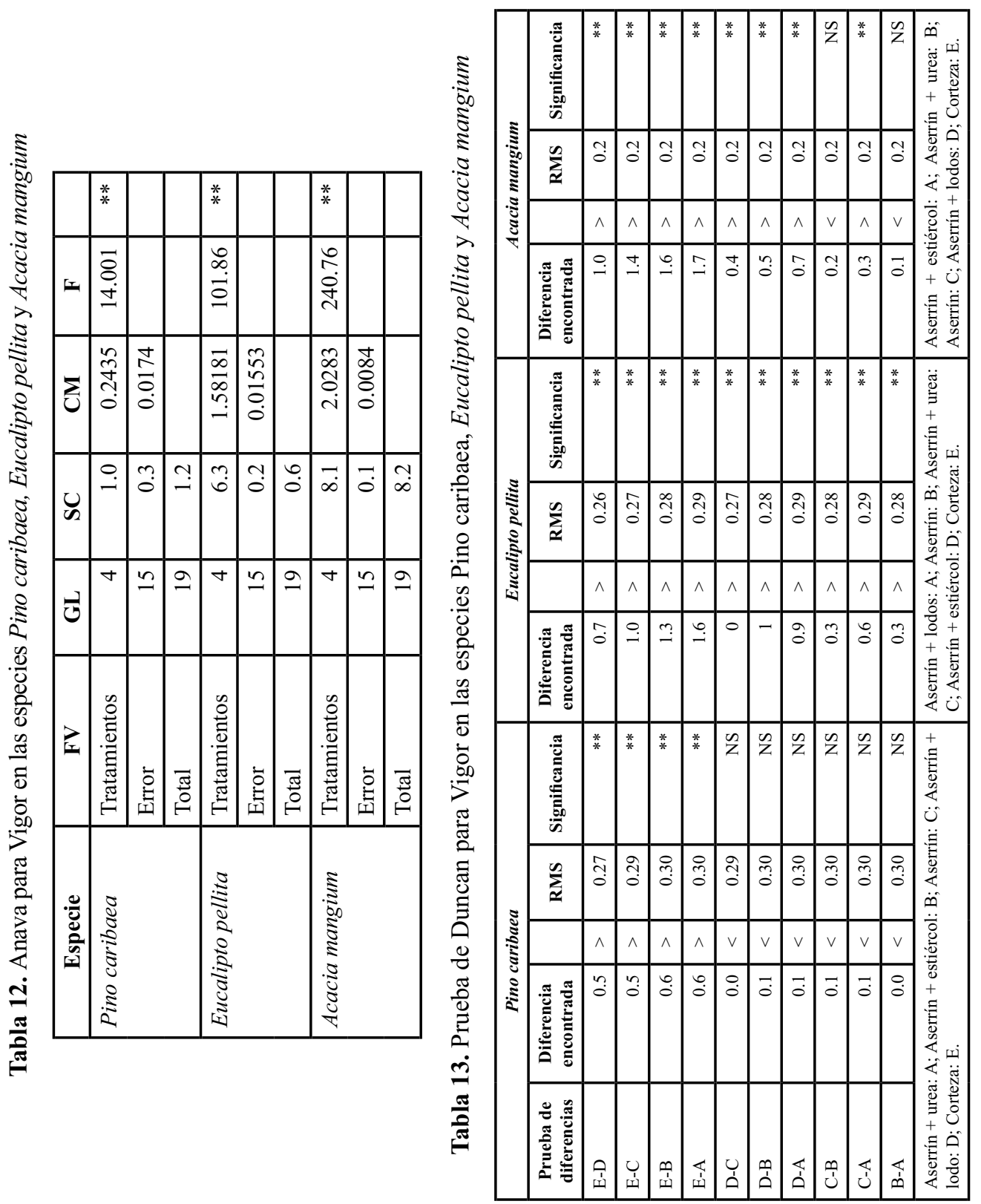


\section{Discusión y Conclusiones}

No obstante el incremento de los costos de producción en los tratamientos de compostaje, el valor agregado a los nuevos productos genera ingresos y reduce costos ambientales de la actividad forestal.

Los mayores impactos ambientales generados por la acumulación de residuos maderables (acumulación de residuos pirófilos; riesgo de incremento de poblaciones insectiles xilófagas en la plantación, bosques y estructuras vulnerables; cambio de características edáficas de los sitios de depósito) pueden reducirse en la medida que se use y se venda este material. Sin embargo, es importante evaluar y mitigar los lixiviados producidos por la aplicación de fuentes nitrogenadas e hidratación continua.

En maíz y Acacia mangium, el porcentaje de germinación presentó tendencias distintas de acuerdo con el sustrato empleado y no se observó toxicidad sobre el material vegetal.

El aserrín sin tratamiento y la corteza fueron para maíz y Acacia mangium, tratamientos poco recomendables para incrementar la biomasa fresca de plantas en germinación.

Los aserrines compostados pueden ser sustratos con potencial para camas germinadoras, con ventajas fitosanitarias sobre sustratos tipo arena. La mayor capacidad de retención de agua disminuiría la necesidad de riego sin afectar la fisiología de la planta.

La corteza de pino compostada, aunque su costo sea significativamente mayor, produjo los mejores resultados en vigor y longitud de tallos de las tres especies forestales. A pesar de ello, se observó en los análisis de tejido vegetal que el contenido en macro y micronutrientes no difiere de los otros tratamientos, por lo que se deberán realizar nuevos análisis de otras variables que puedan interferir en la habilidad de las plántulas para absorber los fertilizantes aplicados foliarmente y en solución.

La variable supervivencia no fue significativa para las especies pino y acacia, pero en eucalipto el tratamiento con mejor tamaño y vigor tuvo menos supervivientes debido a la cantidad de individuos suprimidos dentro de la unidad de muestreo. Por lo anterior se puede concluir que esta especie es altamente heliófila y requeriría el uso de contenedores individuales que permitan la clasificación de los individuos por tamaño.

\section{Recomendaciones}

Se debe probar nuevas dosis de urea, lodos, estiércol y aditivos como la cal para corregir $\mathrm{pH}$, que permitan utilizar los residuos del aserrado de Pino caribaea como sustratos en vivero. El uso no deberá limitarse a la forma pura sino a mezclas que favorezcan el crecimiento de las plántulas de diferentes especies.

Se recomienda ensayar y analizar individualmente la germinación de las especies que se pretendan utilizar con los sustratos utilizados en esta investigación.

Como la fertilización foliar complementa la edáfica, y ante el bajo contenido de nutrientes de los sustratos probados, es aconsejable adicionar abonos de liberación 
lenta que favorezcan el crecimiento de las diferentes especies a ensayar.

El área de palma en Villanueva, Monterrey y Barranca de Upía se incrementó en 10.000 ha, razón que amerita ampliar esta experiencia investigativa a la luz de la utilización de sustratos que reemplacen los grandes volúmenes de suelo requeridos en las etapas de vivero y pre-vivero.

\section{Referencias}

1) Álvarez, E., Díaz, S. \& Alessandrini, M. (2004). Utilización racional de los residuos forestales. Centro de estudios de biomasa forestal. Cuba: Universidad Pinar del Río. Disponible en http://www.biblioteca.org.ar/ libros/88771.pdf.

2) Bámaca F. E., Kanninen. M., Louman, B., Pedroni, L \& Gómez, M. (2004). Contenidodecarbonoen los productos y residuos forestales generados por el aprovechamiento y el aserrío en la reserva de biósfera maya. Recursos naturales y ambiente, comunicación técnica. Cuba: Universidad Pinar del Río. Disponible en http//orton.catie. ac.cr/repdoc/A2185/A2185E.pdf.

3) Bongcam, E. (1982). Estudio detallado de los suelos y uso forestal de las fincas Mata de Humo y La Aurora, Villanueva, Casanare. Bogotá: Compañía Nacional de Reforestación.

4) Maia, C. (1999). Uso da casca de Pinus e lodo biologico como substrato para a produção de mudas de Pinus taeda. Boletim de pesquisa florestal, (39), 81-92. Colombo, Brasil.
5) Midwest Systems. (2008). Turning compost into cash. One farmer's experience in saving and gaining bushels with compost. Composting perspectives, abril, 1-8. Disponible en http://midwestbiosystems.com/ wpcontent/themes/chameleon/ newsletter/newsletter_2008-02.pdf

6) Organizacióninternacionaldemaderas tropicales, ITTO. (2007, 17-19 de mayo). Conferencia internacional sobre dendroenergía llevada a cabo en Hannover, Alemania.

7) Reforestadora de la costa S. A. (2009). Informe de evaluación de certificación del manejo forestal en Municipio de Sabanas de San Ángel (Magdalena) y municipio de Villanueva (Casanare) - Colombia. Disponible en http://info.fsc.org/ servlet/servlet.FileDownload?

8) Reyes, J. (2005). Producción de plántulas de Pinus pseudoestrobus en sustratos a base de aserrín. Chapingo, Serie Ciencias forestales y ambiente. Chapingo, México: Universidad Autónoma de Chapingo.

9) USDA. (s. f.). Forest products laboratory. Forest service: Uses for sawdust, shavings and waste chips.

10)Velásquez, B. (2006). Situación de los sistemas de aprovechamiento de los residuos forestales para su utilización energética. Ecosistemas, Enero-Abril. Alicante, España.

Recibido: 29 de julio de 2011 Aceptado: 30 de septiembre de 2011 